\title{
Reflexión sobre el emprendimiento en el Programa de Administración de Em- presas de la Universidad de Cundinamarca, Chía ${ }^{1}$
}

\section{Reflection on entrepreneurship in the Business Administration Program at Universidad de Cundinamarca, Chía}

\author{
Luz Mireya Rincón Mora ${ }^{2}$ \\ Luz Alejandra Riveros Sáchica ${ }^{3}$ \\ DOI: 10.29151/hojasyhablas.n18a7
}

\section{Resumen}

Este artículo sugiere un mejoramiento en la ruta de formación para el emprendimiento en el programa de Administración de Empresas de la Facultad de Ciencias Administrativas Económicas y Contables de la Universidad de Cundinamarca, Sede Chía. Mediante el empleo de una metodología aplicada, se proponen modificaciones al plan de estudios y cambios en la estructura curricular con el fin de lograr la alineación con los Planes Institucionales y las directrices Gubernamentales; además de un plan para el fortalecimiento del Centro de Innovación, Tecnología y Gestión Organizacinal (CITGO). Para ello, se realizó una encuesta a la comunidad estudiantil y a la comunidad docente sobre emprendimiento, sus implicaciones, sus repercusiones y su percepción con respecto al plan de estudios; a partir de esta información se hace un análisis del plan de estudios, una sugerencia curricular, se analizan las posibles restricciones a la propuesta y se concluye con un análisis de escenarios dada la implementación de la propuesta y el plan de comunicaciones.

Palabras clave: Emprendimiento; reforma curricular; administración de empresas; plan de estudios.
This article suggests an improvement in the training path for entrepreneurship in the Business Administration Program of the Faculty of Economics and Accounting Administrative Sciences at the Universidad de Cundinamarca in Chía. Through an applied methodology, modifications to the curriculum and changes in the curricular structure are proposed in order to achieve alignment with the Institutional Plans and the governmental guidelines, as well as a plan to strengthen the Center for Innovation, Technology and Organizational Management (CITGO in Spanish). A survey was made to the student and the teaching community about entrepreneurship, its implications, its repercussions and its perception with respect to the curriculum. As a result, an analysis of the curriculum and a curricular suggestion is made, as well as the possible restrictions to the proposal, concluding with an analysis of given scenarios for its implementation and its communication plan.

Keywords: Entrepreneurship; curricular reform; business administration; curriculum.

\footnotetext{
${ }^{1}$ Artículo de reflexión resultado de la tesis de grado de Maestría en Organizaciones titulada "Ruta de formación en emprendimiento para el programa de Administración de Empresas en la Universidad de Cundinamarca Sede Chía, 2018

2 Administradora de Empresas. Magíster en Administración de Organizaciones. Directora del Programa de Administración de Empresas, Universidad de Cundinamarca Sede Chía. Fundadora e Investigadora del Grupo de Investigación DOPYS. E-mail: lmireyaricnon@ucundinamarca.edu.co

${ }^{3}$ Psicologa. Magister en Administración de Organizaciones. Docente del Programa de Administración de Empresas, Universidad de Cundinamarca Sede Chía. E-mail: lalejandrariveros@ucundinamarca.edu.co
} 


\section{Introducción}

El emprendimiento es la gran apuesta del futuro y, el fomento del mismo, trae beneficios para la economía de un país, contribuye a los niveles de competitividad, mejora el desempeño económico de una región, genera ingresos complementarios, moviliza socialmente y aporta a las comunidades. (Agarwal, Rahman y Errington, 2009; Amorós y Cristi, 2008; Amorós et al., 2012b; Autio, 2005; Hansson, 2010; OECD, 2003; North y Smallbone, 1996; Petrin, 1997; Poblete y Amorós, 2011; Wennekers et al., 2005; Wong et al., 2005; Mancilla, Amoros, \& Viallant, 2016).

Teniendo en cuenta lo anterior, las universidades no pueden ser indiferentes, por el contrario, su compromiso es contribuir al desarrollo de las regiones y el país, a través de escenarios que lleven a la construcción de conocimiento, innovación y creatividad, basados en componentes estratégicos como la tecnología. El presente trabajo da a conocer una propuesta para el fomento del emprendimiento en el Programa de Administración de Empresas de la Universidad de Cundinamarca, teniendo en cuenta que, para el último registro calificado, tan solo el $16 \%$ de los alumnos en los últimos 5 años, han creado un emprendimiento (Oficina Trabajos de Grado, 2019).

Por tal razón, es importante promover una estrategia que permita contribuir a la consolidación del emprendimiento dentro del Programa, por medio de acciones de mejoramiento y fortalecimiento de sus procesos académicos. Para llevar a cabo dicha estrategia, fue necesario analizar el plan de estudios con el fin de identificar aspectos que contribuyen al emprendimiento, así como a la formación de una cultura emprendedora. A continuación, se presenta el diseño de una línea de formación en la iniciación de nuevos proyectos, y se formula un plan de acción para el Centro de Innovación, Tecnología y Gestión Organizacional (CITGO), unidad que dinamiza el emprendimiento en la Universidad, alineado con documentos institucionales como el Proyecto Educativo Universitario (PEU), el Proyecto Educativo de la Facultad (PEF) y el Proyecto Educativo del Programa (PEP).

La reflexión de este artículo está basada en una encuesta dirigida a docentes y estudiantes del Programa, cada una con 10 preguntas, cuyo fin fue indagar por la percepción frente a la forma como se promueve el emprendimiento en la Universidad. El problema se analizó desde la metodología del árbol de problemas, entendido como una técnica participativa que permite desarrollar ideas creativas y a organizar la información recolectada, generando un modelo de relaciones causa-efecto que explican el problema (Hernández, 2015). Para ampliar las causas del problema formulado, y entender mejor las dinámicas del emprendimiento en la Universidad de Cundinamarca, se realizó otra encuesta dirigida a la totalidad de los docentes (30) del Programa de Administración de Empresas vinculados en modalidades Tiempo Completo Ocasional, Hora Cátedra y Medio Tiempo.

\section{El emprendimiento en la academia}

El término emprendimiento viene del francés entrepreneur (pionero) y hace referencia a la capacidad que tiene una persona para lograr sus objetivos, es decir que, se le llama emprendedor a la persona que tiene actitudes y aptitudes para alcanzar metas y nuevos proyectos. El emprendimiento se conoce como un proceso altamente dinámico, que implica una movilización tanto de pensamiento como de acciones, influido por una posición en un mercado, en el que constantemente se está movilizándo. Así, la perspectiva empresarial está influida por la relevancia que tenga un sector, evaluando varias áreas en varias dimensiones, que llevan a evaluar a una posición, a tomar decisiones y pasar a la acción (Herrera y Montoy, 2013, p. 53). 
En cuanto a la vinculación entre academia y emprendimiento, varios autores han señalado su relación. Para Sanabria-Rangela, Morales-Rubiano y Ortiz-Riagac (2015), desde la intención emprendedora formada por la misma universidad, así como dentro de los conceptos que se engloban como Universidad Emprendedora; otros autores afirman que la academia tiene una incidencia fuerte en el desarrollo de los emprendimientos y pueden promover programas con un rol más activo (Castelao, González, Jordán y Ruiz, 2015; Azanza y Campos, 2014; Arroyo, 2016; HervásOliver, Boronat-Moll y Messana, 2017; Allen y O'Shea, 2014). Finalmente, se ha hecho énfasis en la creación de una estrategia de emprendimiento, a partir del fortalecimiento del proceso de extensión universitaria (Sanabria-Rangel, MoralesRubiano y Ortiz-Riaga, 2015); así, hablar del emprendimiento trae implícito el valor agregado que le da la universidad al egresado, en términos de capacidad para dinamizar procesos, servicios o productos (Romero y Núñez, 2014).

Soto y Forero (2016) realizan una prospectiva para el sector educativo en Colombia, en la cual la utopía de la Universidad se contradice con la realidad del mercado, llegando a la conclusión de que falta una verdadera relación entre las TIC las necesidades sociales y la formación humanística. No basta con tener un currículo orientado al emprendimiento, sino que se requieren recursos para el aprendizaje, docentes debidamente capacitados y con habilidades para la investigación, conduciendo a los integrantes de la comunidad educativa en las tendencias del mercado y en la creación, conformación y puesta en marcha de emprendimientos viables en el mercado (Martínez, Tobón y López, 2018).

De igual manera, Murcia, Morales y Ramírez (2015) señalan que no es suficiente tener un plan de estudios orientado al emprendimiento, sino se debe propender por una coherencia entre los discursos de diferentes materias y rutas de formación articulados con la generación de conocimiento, creación de empresas y emprendimientos. Y como refiere Moncada (2010, citado en Montaña, 2016) se hace necesario generar instituciones educativas que permitan a las sociedades desarrollar cualidades en competencias, capacidades y destrezas, respondiendo a las exigencias del mundo actual ávido de Estados generadores de desarrollo, emprendimiento y transformación.

También es importante identificar elementos del emprendimiento en algunas universidades colombianas, pues esto servirá de referencia para sustentar el estudio y la propuesta de modificación curricular.

Es por ello que, a la Universidad de Cundinamarca le corresponde actuar como coadyuvante del proceso de emprendimiento, lo potencialice, lo fomente y desarrolle desde los programas académicos, así como lo evidencie desde el Proyecto Educativo Universitario (PEU), el Proyecto Educativo de Programa (PEP) y la planeación estratégica de la Universidad, buscando una articulación que permita una integralidad en el proceso formativo hacia el emprendimiento. A continuación, se presenta un plan de acción que apoya las propuestas de este estudio y que busca identificar la mejor solución en términos de impacto y efectividad. 
Tabla 1. Emprendimiento en las Principales universidad colombianas

\begin{tabular}{|c|c|}
\hline UNIVERSIDAD & ENFOQUE \\
\hline Universidad de Los Andes & $\begin{array}{l}\text { Formar profesionales con competencias globales y de } \\
\text { liderazgo, que se vinculen a actividades de } \\
\text { emprendimiento, empresas, organizaciones públicas, } \\
\text { tercer sector y a la comunidad, con una clara vocación de } \\
\text { agentes de cambio en la sociedad colombiana. }\end{array}$ \\
\hline Universidad Nacional de Colombia & $\begin{array}{l}\text { Cuentan con Unidad de Emprendimiento e Innovación, } \\
\text { Colombia Star-up. }\end{array}$ \\
\hline Universidad Javeriana & $\begin{array}{l}\text { Proyecto que busca alinear los esfuerzos de las } \\
\text { Facultades en torno a la formación del perfil } \\
\text { emprendedor en los egresados de la Universidad. }\end{array}$ \\
\hline Universidad del Rosario & $\begin{array}{l}\text { Cuentan con un Centro de Emprendimiento, además de } \\
\text { una estrategia curricular, menciones en emprendimiento, } \\
\text { opción de grado en emprendimiento y práctica en } \\
\text { emprendimiento. }\end{array}$ \\
\hline Universidad Externado de Colombia & $\begin{array}{l}\text { Diplomado en emprendimiento y apoyo a estudiantes de } \\
\text { pregrado y posgrado. }\end{array}$ \\
\hline $\begin{array}{l}\text { Universidad EAN (Escuela de Administración } \\
\text { de Negocios) }\end{array}$ & $\begin{array}{l}\text { Política y enfoque hacia el emprendimiento sostenible, } \\
\text { formación transversal, conformación del Instituto para el } \\
\text { emprendimiento sostenible, capital para inversión. }\end{array}$ \\
\hline $\begin{array}{l}\text { CESA (Colegio de Estudios Superiores de } \\
\text { Administración) }\end{array}$ & Centro de Innovación y Emprendimiento. \\
\hline Universidad de la Sabana & $\begin{array}{l}\text { Semillero en Emprendimiento e Innovación Sabana. } \\
\text { Modelo de acompañamiento: agilismo para } \\
\text { emprendedores, Acompañamiento de incubación a } \\
\text { emprendimientos, Programa Forma Emprende, } \\
\text { Acompañamiento opción de práctica en emprendimiento, } \\
\text { Convocatoria Generación Seis }\end{array}$ \\
\hline Universidad Militar Nueva Granada & Centro de Innovación y Emprendimiento Neogranadino. \\
\hline Universidad Manuela Beltrán & CREAR, la incubadora de empresas de la UMB. \\
\hline
\end{tabular}

Fuente: Elaboración propia 


\section{Tabla 2. Plan de acción para la Universidad de Cundinamarca}

\begin{tabular}{|c|c|}
\hline \multicolumn{2}{|c|}{$\begin{array}{l}\text { 1. Análisis y fortalecimiento de la estructura curricular del plan de estudios del Programa de } \\
\text { Administración de Empresas desde la mirada del emprendimiento }\end{array}$} \\
\hline META & INDICADOR \\
\hline $\begin{array}{l}\text { Diseño de nueva ruta de emprendimiento a través } \\
\text { de una propuesta curricular }\end{array}$ & $\begin{array}{l}\text { Creación de nuevos espacios } \\
\text { emprendimiento }\end{array}$ \\
\hline \multicolumn{2}{|c|}{ 2. Fortalecimiento del centro de Innovación, Tecnología y Gestión Organizacional } \\
\hline META & INDICADOR \\
\hline Diseño de un plan de acción para el CITGO & Plan de acción \\
\hline \multicolumn{2}{|c|}{ 3. Estrategias para la creación de cultura orientada al emprendimiento } \\
\hline META & INDICADOR \\
\hline $\begin{array}{l}\text { Definición de estrategias orientadas a la } \\
\text { consolidación de una cultura emprendedora }\end{array}$ & $\begin{array}{l}\begin{array}{l}\text { Estrategias } \\
\text { planeadas }\end{array} \\
\text { desarrolladas vs estrategias }\end{array}$ \\
\hline
\end{tabular}

Fuente: Elaboración Propia

\section{Retos desde la Universidad}

El impacto de las soluciones planteadas será evidenciado de manera significativa con las cohortes de estudiantes, con ingreso posterior a la implementación de la propuesta, donde se espera que los indicadores de estudiantes emprendedores en el Programa de Administración de Empresas se incremente de manera considerable y cuenten con la seguridad, así como con las habilidades que les permita alcanzar de manera exitosa la meta propuesta.

Siguiendo esta línea, es importante reflexionar frente a las "reformas curriculares o educativas que escribamos en los papeles, [porque] somos las personas las que hacemos los cambios y no los decretos"; por tanto, es importante capacitar y entrenar al equipo docente para que ellos formen estudiantes innovadores y emprendedores con las competencias y habilidades que coadyuven a asumir retos en un mundo dinámico y cambiante.
De este modo, para fomentar el emprendimiento en el programa de Administración de Empresas es fundamental contar con una ruta de formación orientada al fortalecimiento de ideas emprendedoras, donde el punto de partida sea un diagnóstico que identifique los conocimientos y habilidades iniciales que tiene el emprendedor, seguido de espacios académicos que brinden asesoría y acompañamiento durante el proceso que conduzca a la madurez del proyecto. Entonces, la ruta propuesta la conforman siete núcleos de aprendizaje que son: cultura emprendedora, emprendimiento e innovación, gestión y prototipado, investigación de mercados, diagnóstico organizacional, formulación y evaluación de proyectos y gestión de proyectos.

Así mismo, se requiere dar mayor fortalecimiento al Centro de Innovación Tecnología y Gestión Organizacional (CITGO). A pesar de no contar con una política explícita de emprendimiento, la Universidad de Cundinamarca sí tiene consignados en sus documentos institucionales la importancia de promover el desarrollo de 
cultura emprendedora y de ideas de negocios.

Considerando los argumentos de Romero (1999), "el espíritu empresarial está muy unido a la motivación al logro" (p. 25) y la pretensión del programa es desarrollar una cultura emprendedora que aporte al tejido empresarial; por tanto, la estructura curricular debería contar desde los primeros momentos de formación con núcleos temáticos, que brinden nuevos saberes en torno al emprendimiento y a su vez desarrolle competencias y habilidades encaminadas a potenciar actitudes emprendedoras que pueden llevar al nacimiento de una empresa o a la formación de valores personales con un compromiso innovador y trascendente.
Teniendo en cuenta la malla curricular implementada desde 2017, se evidencia que el Programa no cuenta desde los inicios con un proceso de enseñanza-aprendizaje con espacios académicos que desarrollen y fortalezcan en los educandos valores y competencias emprendedoras. Por ejemplo, en tercer semestre se tiene un núcleo temático denominado proyecto de empresa, a través del cual se lleva al estudiante a generar ideas de negocio, según lo establecido en el syllabus, abordando la educación empresarial en su conjunto.

\section{Ilustración 1. Plan de Estudios Vigente 2013-2019}

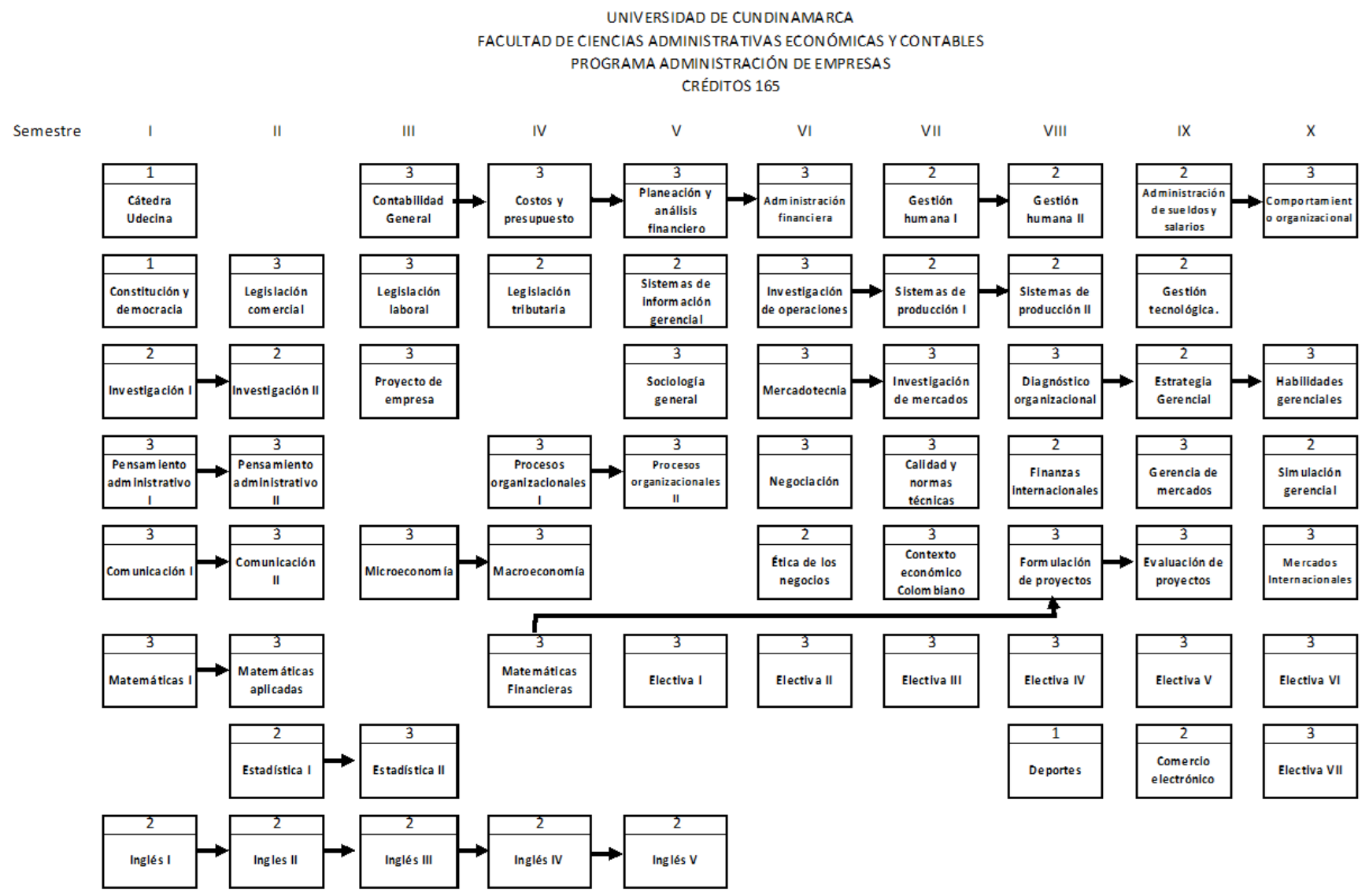

Fuente: Universidad de Cundinamarca 
De cuarto a décimo semestre, el plan de estudios cuenta con tres núcleos temáticos que, de manera directa, intervienen en el proceso de formación empresarial y específicamente en la creación de empresa, tal como se evidencia en la siguiente ilustración:

\section{Ilustración 2. Plan de estudios 2013-2019}

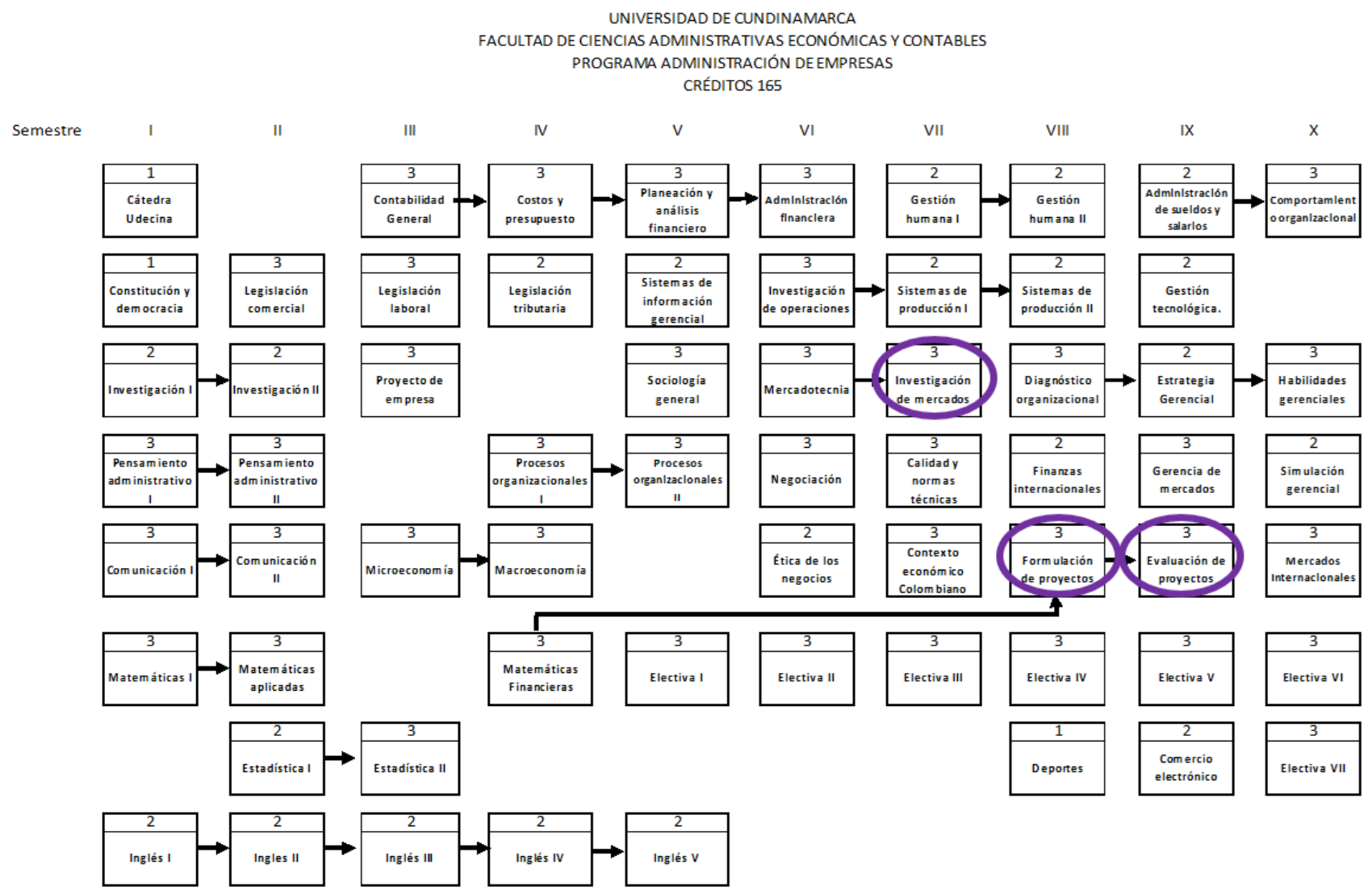

Fuente: Universidad de Cundinamarca

Como se puede observar, existe un núcleo de investigación de mercados en séptimo semestre, la formulación de proyectos en octavo y la evaluación de proyectos en noveno; es decir que, en cuarto, quinto, sexto y décimo semestre no hay núcleos temáticos que contribuyan de manera directa o aporten al proceso de formación en emprendimiento, siendo claves al brindar elementos conceptuales sólidos que permiten a cada estudiante estructurar proyectos viables y sostenibles.

Es de anotar que, los demás núcleos temáticos que conforman el plan de estudios no se orientan a la formación del perfil profesional anhelado, sin que esto implique que todos los estudiantes tengan que ser empresarios, sino más bien que tengan capacidades para desempeñarse de manera estratégica e innovadora en una empresa, es decir, sean "intraemprendedores" (Vega, 2018; Morris et al., 2011; Bosma et al., 2010; Veenker, Sijde, During y Nijhof, 2008). Por el contrario, en los syllabus se evidencia que los contenidos, las herramientas y las metodologías propuestas están alejadas de la propuesta institucional en emprendimiento, pues solamente se centran sobre el plan de negocios, estudios de mercado, y no se profundiza en las herramientas que debe tener un intraemprendedor.

De otro lado, analizando el proceso final de formación concluido en la opción de grado, donde 
los estudiantes cuentan con siete posibilidades que son: pasantía, investigación, proyecto monográfico, semestre avanzado, proyección social, restructuración empresarial y emprendimiento; aquella con menor índice de postulación es el emprendimiento, con una participación aproximada del $2 \%$ del total de las opciones de grado (Opciones de Grado Chía, 2014-2018).

En síntesis, se puede decir que el actual plan de estudios del Programa de Administración de Empresas de la Universidad de Cundinamarca ob- staculiza el logro del perfil profesional propuesto, y su propósito de formación ya que no se presenta de manera secuencial con la formación y el aprendizaje en conocimientos, capacidades, habilidades y competencias emprendedoras (Denner, 2012; Briscoe y Rogan, 2016; Luna y Velasco, 2017).

De esta manera, y tratando de resolver dicha falencia, la propuesta del nuevo plan de estudios para el Programa de Administración de Empresas de la Universidad de Cundinamarca, es la siguiente:

\section{Ilustración 3. Plan de Estudios del Campo de Aprendizaje Emprendimiento e Innovación}

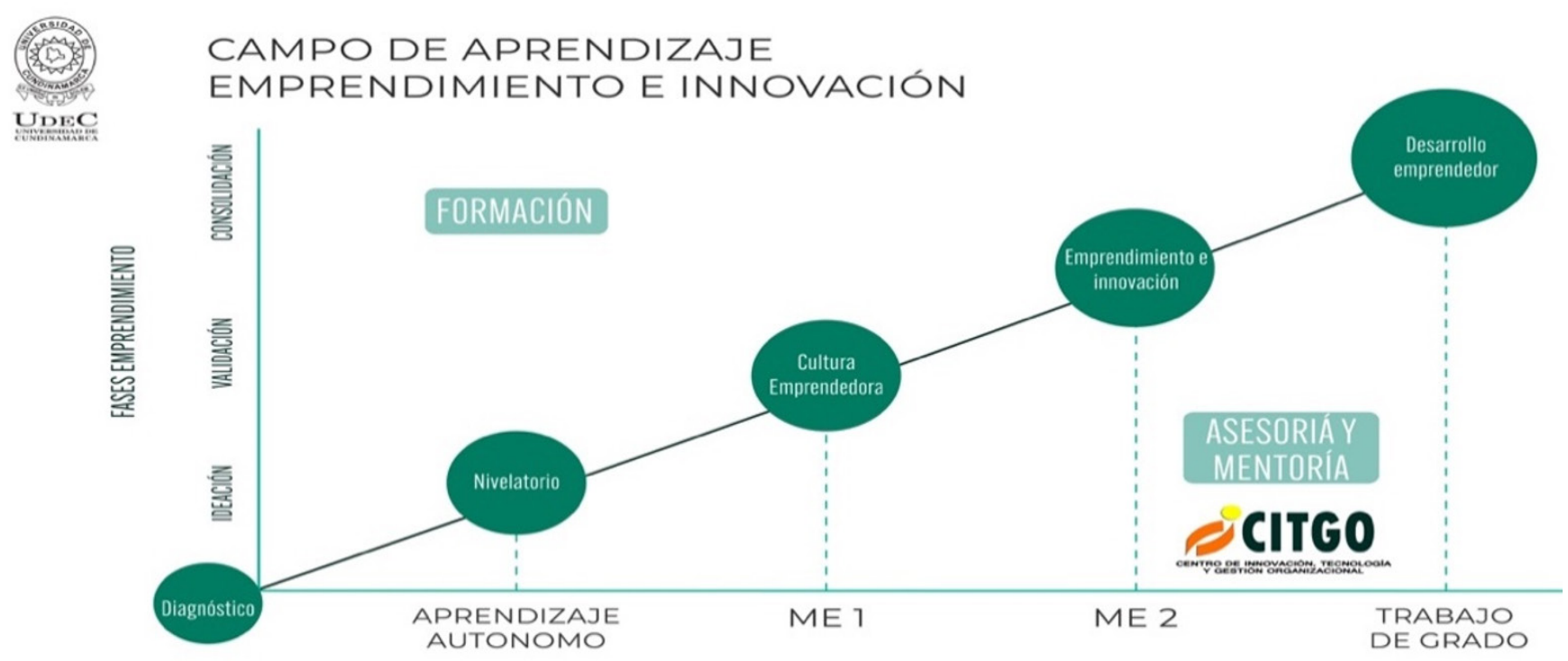

El nuevo Modelo Pedagógico Digital Transmoderno de la Universidad de Cundinamarca MEDIT incorpora elementos digitales dentro del proceso de formación y construye una relación más vertical, en la que el alumno genera su proceso de aprendizaje con el acompañamiento del docente (Quintero, 2019). Bajo esta óptica, nace el concepto de Campo de Aprendizaje Institucional, que es el abordaje transversal de elementos comunes entre los integrantes de la comunidad educativa, entre los que se encuentra el Campo de Aprendizaje de Emprendimiento e Innovación (en adelante CAIEI).
El CAIEI es un escenario multidimensional que consolida las relaciones Universidad-Comunidad-Empresa-Estado, y busca la generación y el fortalecimiento de la cultura emprendedora e innovadora, por medio del crecimiento en cuatro momentos de formación: nivelación, cultura emprendedora, emprendimiento e innovación, desarrollo y crecimiento emprendedor, las cuales se desarrollan en dimensiones como el aula, la familia, la institución, la persona, la naturaleza, la cultura y la sociedad. El proceso estará articulado de manera transversal con el CITGO (Centro de Innovación Tecnología y Gestión Organizacional) quien brindará asesorías, mentorías y capacitaciones a la comunidad (Documento CAIEI, 2019). 
Ilustración 4. Propuesta Ruta de Emprendimiento Programa de Administración de Empresas
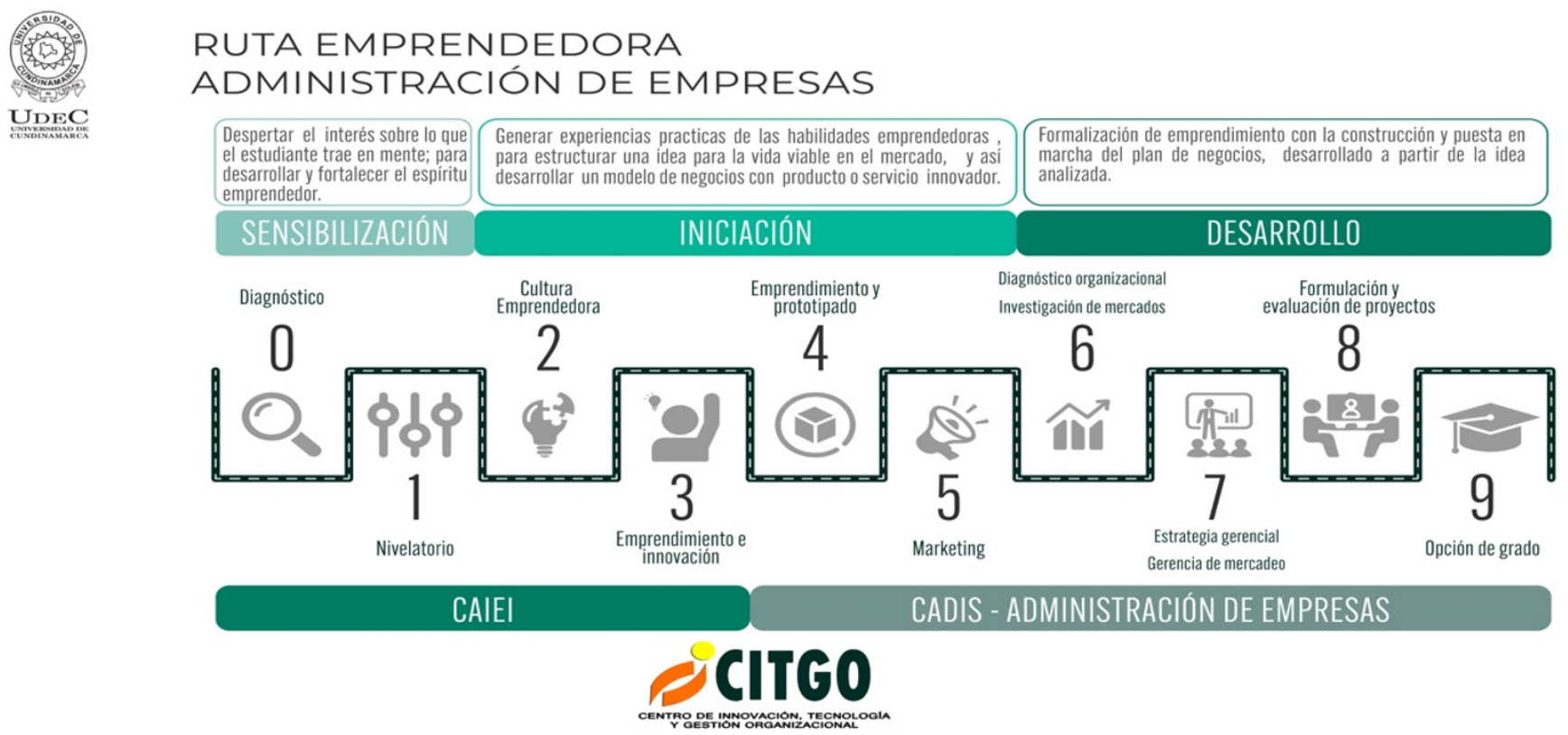

Como se puede evidenciar en la Ilustración 4, el CAIEI abarca los tres primeros semestres, a partir de cuarto semestre se articula con los Campos de Aprendizaje Disciplinares (CADIS) de emprendimiento y prototipado, marketing, diagnóstico organizacional, investigación de mercados, estrategia gerencial, estrategia de mercadeo y formulación, y evaluación de proyectos para finalizar con la opción de grado en emprendimiento.

\section{Conclusiones}

El emprendimiento es uno de los principales derroteros de las universidades actualmente en Colombia. La mayoría de Instituciones de Educación Superior realiza acciones, planes y proyectos para promover e incentivar las ideas de negocio. La articulación entre la Academia, el Estado, y el sector productivo permite fortalecer las ideas y proyectos de los emprendedores, generando impacto en el crecimiento del tejido empresarial regional. Por tanto, es necesario que la Universidad de Cundinamarca propicie alianzas estratégicas que conduzcan al fomento del emprendimiento en la Institución.
De igual manera, se requiere la formación en emprendimiento de todos los docentes para que dentro de cada uno de sus núcleos temáticos alienten la formación de nuevas ideas de negocio, logrando así una articulación entre toda la comunidad educativa de la Universidad de Cundinamarca.

Es por ello que, es perentorio fortalecer al CITGO con perfiles de liderazgo, programas y conformación de redes para que la Comunidad Udecina y con visibilidad en las diferentes instancias que apoyan el emprendimiento a nivel local, nacional e internacional; y es fundamental que el estudiante de Administración de Empresas de la Universidad de Cundinamarca obtenga en su proceso de formación las competencias y saberes necesarios para desarrollarse como un emprendedor exitoso, mediante el desarrollo del plan de estudios propuesto y la puesta en marcha de la idea emprendedora, porque el emprendimiento es en sí mismo una mecanismo de ubicación laboral y de transformación económica y social. 


\section{Referencias bibliográficas}

Allen, T. y O’Shea, R. (2014). Building Technology Transfer within Research Universities. United Kingdom: Cambridge University Press.

Arroyo, M. (2016). Emprendimiento y Universidad Emprendedora: conceptualización, propuesta metodológica y caracterización de la Universidad Politécnica de Valencia. Valencia: Universidad Politécnica de Valencia.

Azanza, G. y Campos, J.A. (2014). La intención emprendedora en estudiantes universitarios: el caso de la universidad de deusto. Boletín de estudios económicos, LXIX (211), p.p. 151-172.

Castelao, M.; González, J.; Jordán, A.; y Ruiz, A. (2015). Universidad y emprendimiento. Intención emprendedora en estudiantes de universidades privadas madrileñas. Revista de Investigación en Educación, 2 (13), p.p. 187-205.

Herrera, C. y Montoya, L. (2013). El emprendedor: una aproximación a su de nición y caracterización. Punto de Vista, IV(7), p.p. 7-30.

Hervás-Oliver, J.; Boronat-Moll, C.; y Messana, I. (2017). La Universidad Española como plataforma de emprendimiento: Hacia la Universidad Emprendedora del Futuro. Economía Industrial. (404), p.p. 11-19.
Mancilla, C.; Amoros, E.; y Viallant, Y. (2016). Análisis de las primeras etapas del proceso emprendedor. Universidad de los Lagos, p.p. 13-22.

Martínez, J.; Tobón, M.; y López. (2018). Acreditación de la calidad en instituciones de educación superior: retos pendientes en América Latina. Revista Dilemas Contemporáneos: Educación, Política y Valores, 1-20.

Montaña, C. (2016). Desafíos de la educación superior colombiana frente a las expectativas del mercado y la internacionalización. Universidad Militar Nueva Granada.

Murcia, C.; Morales, A.; y Ramírez, J. (2015). Formación para el emprendimiento en estudiantes de Administración de Empresas. Educación Humanismo. DOI https://doi. org/10.17081/eduhum.17.28.1166

Romero, y Nuñez. (2014). Universidades con valor añadido: empleabilidad y emprendimiento innovador. Procedia - Social and Behavioral Science, p.p. 65-71.

Sanabria-Rangel, P.; Morales-Rubiano, M.; y Ortiz-Riaga, C. (2015). Interacción universidad y entorno: marco para el emprendimiento. Educación y Educadores, p.p. 111-134.

Soto, D. y Forero, A. (2016). La Universidad Latinoamericana y del Caribe en los desafíos del Siglo XXI. Rev. historia de la educación latinoamericana, 18 (26), p.p. 279-309. 